\title{
Sleepless in the surgical ward
}

$\infty \quad$ See related editorial, page 1087

$\mathrm{B}$ abies love it, workaholics hate it, coffee-drinkers delay it, but none can avoid it. All living creatures need sleep. There are few things that degrade human performance, mental or physical, as much as forgoing sleep for extended periods. And if you are chronically sleep deprived, not only will your performance suffer, you could, depending on your profession, be putting yourself and others at risk.

That's why many professionals long-haul truckers, nuclear technicians, commercial airline pilots must follow rules limiting the lengths of their shifts.

"For reasons that are unclear to me, medicine doesn't have any of those rules," says Dr. Najib Ayas of Vancouver Coastal Health's Sleep Disorders Program in Vancouver, British Columbia.

Many doctors put in incredibly long hours, but medical residents in particular are expected to sacrifice sleep for work. It is not uncommon for surgical residents, for instance, to spend more than 100 of a week's 168 hours in hospital.

In Canada, medical residents routinely put in extended shifts of 30 hours or more, even though going an entire day without sleep decreases performance as much as having a 0.10 bloodalcohol level. In the 1990s, during his internal medicine residency in the United States, Ayas once worked 40 hours straight.

For decades, researchers have shown that a lack of shut-eye takes a toll on residents and their patients. As a result, many countries have dramatically reduced resident work hours, but Canada and the United States are exceptions. Yet even North American sleep experts, like Ayas, warn that the solution to the sleepy-resident problem may not be as simple as restricting work hours.

"To a certain extent, medicine is an experiential type of learning," says Ayas, who is also a professor of medi-

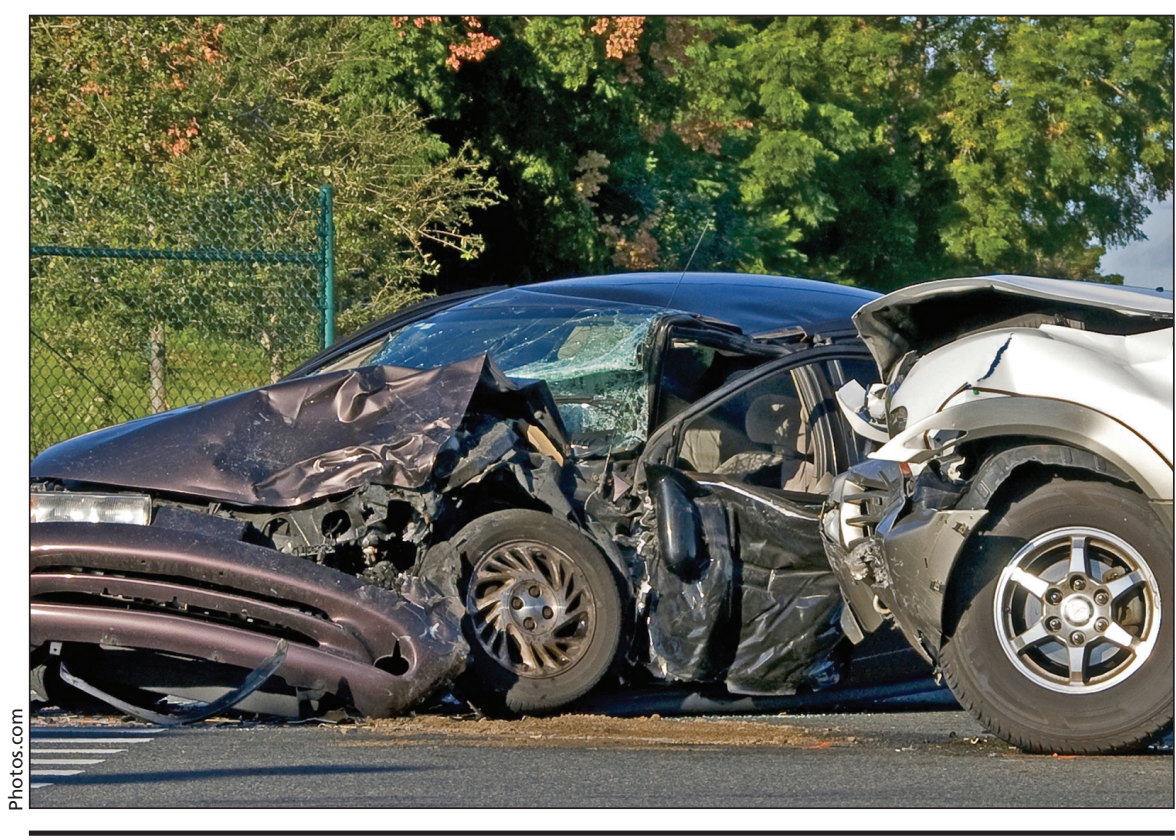

Medical residents are prone to motor vehicle crashes after extended shifts, and are twice as likely to make major medical errors near the conclusion of a shift, according to research conducted by Dr. Najib Ayas of Vancouver Coastal Health's Sleep Disorders Program (CMAJ 2008;179[12]:1254).

cine at the University of British Columbia. "If you reduce the number of hours, are you diluting clinical experience? No one really knows the answer to that question."

In December 2008, the US Institute of Medicine recommended that the Accreditation Council for Graduate Medical Education, which accredits most of the 8500 US residency programs, adjust the workload of medical residents. The accrediting body had already set a national maximum of 80 -hour workweeks in 2003.

Now the Institute of Medicine suggests the accreditation council should also restrict the maximum shift length to 16 hours, down from the current 30 hour limit.

There is considerable evidence to back the Institute's claims that extended shifts are best avoided. A 2004 study found that residents made $35.9 \%$ more serious medical errors and 5.6 times as many serious diagnostic mistakes when working traditional sched- ules instead of ones that eliminated shifts of 24 hours or more (NEJM 2004;351:1838-48). Ayas coauthored a 2005 study that indicated residents are more than twice as likely to have a motor vehicle crash while commuting home after an extended shift, as compared to a regular shift, and are 5 times as likely to experience a near-miss incident (NEJM 2005;352:125-34).

Post-call residents who cause harm to others on the commute home could also face legal problems. In the United States and Britain, drivers impaired by fatigue have been convicted of vehicular homicide.

There are 4 physiological factors that contribute to alertness - circadian phase (time of day), number of hours awake, sleep duration and sleep inertia (time needed to overcome grogginess after waking) - and extended shifts mess with all of them. Furthermore, an estimated $25 \%$ of people are especially sensitive to sleep loss, and residents, of course, are no exception. 
Other research suggests fatigue often plagues the resident population, hampering learning, warping risk assessment and, in some cases, leading to mood disorders, substance abuse and marital problems.

The problem of too-long hours for doctors has largely been addressed in Europe, where the European Union restricts physicians' shifts to 13 hours and their workweeks to 48 hours. In France, doctors work a maximum of 52.5 hours a week, and in Denmark, only 37 hours. Weekly work-hour limits in the United Kingdom range from 56 to 64 hours.

In Canada, rules about work hours are set provincially and laid out in the collective agreements of residency associations. Most provinces don't set a weekly limit on hours, though there are exceptions such as Manitoba (89 hours) and the Maritime provinces (95 hours).

The Professional Association of Internes and Residents of Ontario recently changed its post-call rules, which now state that residents working overnight begin handing over patients at $8 \mathrm{am}$ and leave the hospital by 10 am. Still, some residents claim that the language in collective agreements is often ambiguous and there are too many exceptions, and that rules are not enforced, nor compliance monitored. In the end, they say 30-hour shifts remain commonplace.

"Thirty hours in a row is probably too much. What the right amount is, that's difficult to know," says Dr. Laine Green, a neurology resident at Dalhousie University in Halifax, Nova Scotia, and president of the Professional Association of Resident Physicians in the Maritime Provinces. "Residents don't want to avoid call shifts, because there is valuable learning that occurs when on call."

In 2007, UBC researchers surveyed first-year residents and found that those in surgical rotations worked an average of 80.4 hours a week, while nonsurgical residents worked an average of 57 hours (BCMJ 2007;49[7]:387-92). Doctors who were married or had children worked fewer hours than single, childless physicians. But fewer hours at work didn't necessarily equate to more sleep. The survey found that while male

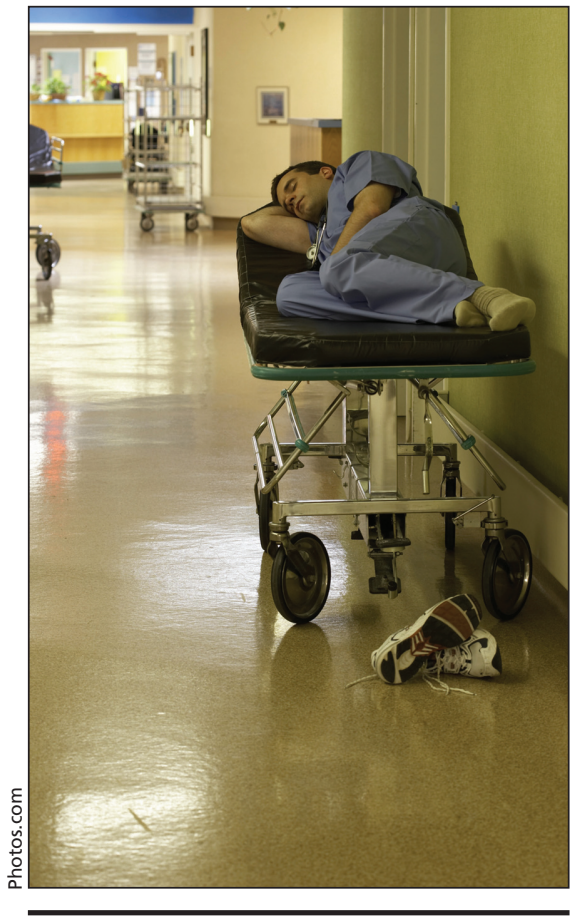

Although many professions have limited the lengths of shifts, medical residents in Canada routinely toil for 30 or more consecutive hours.

doctors worked 5 hours more than their female colleagues each week, they slept 2 hours less.

Though research suggests doctors should get more sleep, and some medical bodies try to regulate their schedules, many residents feel they have little choice but to work long hours, says Dr. Andrea Townson, clinical assistant professor in UBC's Division of Physical Medicine and Rehabilitation.

"The medical culture has been to work long hours, and to celebrate that," says Townson, coauthor of the study. "There is sort of a hero culture. You work long hours, you don't sleep much, and you get a lot of work done."

On the surface, she says, limiting resident work hours appears to make sense, but more research is needed to prove it makes sense. There is a particular need for more Canadian data because subtle differences may exist between the work habits of residents in Canada and the United States, where most of the research in this area is conducted. That's not to say that Canadian residents don't realize fatigue leads to errors, says Townson, but awareness doesn't make it any easier to balance educational, physiological and patient needs.

"There are studies out there that show medical trainees pay a price," says Townson. "I think you do pay a price, but how do we cost that price?"

Another problem with reducing resident work hours is that it would result in more patient handovers, which are significant points of error. Hospitals operate on 24-hour rounds, and if residents worked shorter shifts, communication between incoming and outgoing doctors would have to improve or mistakes could increase, no matter how alert the staff.

Some residents also worry that shorter workweeks would translate to a longer residency. And, of course, there's the unavoidable money issue. If residents work shorter hours, hospitals will need more residents.

The Institute of Medicine estimates that it would cost US $\$ 1.7$ billion annually to meet its recommendations, and that US hospitals would have to hire more than 8000 additional residents.

"The ideal is based on principles, not numbers," says Dr. Mark Masterson, an anesthesiology resident and president of the Professional Association of Residents of British Columbia. "It would be an environment where you get enough exposure and training, but you aren't overworked and it's still safe for patients."

Some hospitals, instead of reducing work hours, have made other moves to address the effects of resident fatigue. For example, the Department of Surgery at the University of Michigan in Ann Arbor, Michigan offers taxi vouchers to residents coming off extended shifts.

Other initiatives include designing schedules that are less disruptive to natural sleep cycles, restricting "medical moonlighting" (additional paid work) and reducing work of little educational value.

"I'm hopeful that things will change for the better," says Masterson. "There is starting to be a cultural change throughout medicine, with the younger generation of physicians recognizing that wellness and balance are important." - Roger Collier, CMAJ

DOI: $10.1503 / \mathrm{cmaj} .090772$ 